Tohoku J. Exp. Med., 2004, 204, 221-228

\title{
Cervical Cytologic Examination During Physical Checkup of Pregnant Women: Cervical Cancer Screening in Women Under the Age of Thirty
}

Yoriko Abe, Kiyoshi Ito, Chikako Okamura, Hitoshi Niikura, Yukiniro Terada, Takashi Murakami, Tadao Takano, Nobuyoshi Ozawa, ${ }^{1}$ Hiroshi Nagai, ${ }^{1}$ Yuichi Wada, ${ }^{2}$ Shigeki Uehara, ${ }^{3}$ Takanori Watanabe, ${ }^{4}$ Shingo Tanigawara, ${ }^{5}$ Shigesato Wagatsuma, ${ }^{6}$ Yuji Yoshida, ${ }^{7}$ Tsukasa Igarashi, ${ }^{8}$ Masakuni Suzuki, ${ }^{9}$ Kimio Nakagawa, ${ }^{10}$ Kunihiro OKamura and Nobuo Yaegashi

Department of Obstetrics and Gynecology, Tohoku University, Sendai, ${ }^{1}$ Miyagi Prefecture Branch, Japanese Medical Association of Obstetrics and Gynecology, Sendai, ${ }^{2}$ National Sendai Hospital, Sendai, ${ }^{3}$ Tohoku Kousai Hospital, Sendai, ${ }^{4}$ Sendai Municipal Hospital, Sendai, ${ }^{5}$ Sendai Red Cross Hospital, Sendai, ${ }^{6}$ Furukawa Municipal Hospital, Furukawa, ${ }^{7}$ Sanuma Municipal General Hospital, Sanuma, ${ }^{8}$ Miyako Prefectial Hospital, Miyako,

${ }^{9}$ Suzuki Hospital, Iwadeyama, and ${ }^{10}$ Nakagawa Obstetrics and Gynecology Hospital, Furukawa, Japan

Abe, Y., Ito, K., Okamura, C., Nitkura, H., Terada, Y., Murakami, T., Takano, T., Ozawa, N., Nagai, H., Wada, Y., Uehara, S., Watanabe, T., Tanigawara, S., Wagatsuma, S., Yoshida, Y., Igarashi, T., Suzuki, M., Nakagawa, K., Okamura, K. and Yaegashi, N. Cervical Cytologic Examination During Physical Checkup of Pregnant Women: Cervical Cancer Screening in Women Under the Age of Thirty. Tohoku J. Exp. Med., 2004, 204 (3), 221-228 — Recently, the Ministry of Health, Labor and Welfare proposed that cervical cancer screening should be conducted for women aged 20 to 29 years old in Japan. However, there are insufficient data available in Japan concerning the screening conducted for women under the age of 30. Therefore, we made a survey of the results of cervical cytologic examination for pregnant women. 28616 pregnant women were examined as subjects of a study group. A group of 108289 women, subjected to group screening for cervical cancer in Miyagi Prefecture, were studied as a control group. The rate of subjects who required close examination in the pregnant women's group was significantly higher than that in the mass screening group $(1.12 \%$ vs. $0.84 \%)$. The rate of close examination was significantly higher in the women 19 years old or younger compared to those in the age group of 25 to 39 years old. The rate was also significantly higher in women aged 20 to 24 years old than those who are 25 to 34 years old. Of the 321

Received June 24, 2004; revision accepted for publication September 14, 2004.

Address for reprints: Kiyoshi Ito, Department of Obstetrics and Gynecology, Tohoku University, 1-1 Seiryomachi, Aoba-ku, Sendai 980-8574, Japan.

e-mail: kito@mail.tains.tohoku.ac.jp 
subjects who required close examination, 34 cases underwent treatment, and 17 cases were under age 30. Moreover, all three cases of microinvasive and/or invasive carcinoma were under the age of 30 years $(23,23,27$ years old, respectively). Our results suggest that screening for cervical cancer in pregnancy is a useful means to find cervical neoplasia in young women and is effective in reducing the cervical cancer morbidity rate. - cervical cancer; examination; pregnancy; young women; mass screening

(C) 2004 Tohoku University Medical Press

In Japan, cervical cancer screening is conducted as a national project under the Health and Medical Service Law for the Aged, and early discovery has contributed to reduction of morbidity and mortality rates for cervical cancer. A study by the Ministry of Health, Labor and Welfare evaluating the effectiveness of cancer screening concluded that "there is sufficient evidence validating the effectiveness of cervical cancer screening by cytologic examination in women aged 30 or older" (Japan Public Health Association 2001).

On the other hand, the current system of cervical cancer screening has several problems. The first one is the low screening rate. For example, the rate of subjects undergoing cervical cancer screening in Miyagi Prefecture is the second highest in Japan (Health and Welfare Statistics Association 2000). Yet, this screening rate does not exceed $30 \%$ of the population (Sato et al. 1998; Miyagi Cancer Society 2003). In addition, the rate of subjects undergoing initial screening is only $10 \%$ of the total number of examination subjects (Sato et al. 1998; Miyagi Cancer Society 2003), although more than two thirds of cervical cancer cases are discovered in the initial screening. The second problem is the age of the subjects, i.e., current screening is limited to subjects aged 30 or older. In recent years, there has been an increase in incidence of cervical cancer among young women in Japan. While the overall morbidity rate of cervical cancer has been declining, the morbidity rate in the 20's and 30's age groups has been rising (Wright et al. 1994; Hamasaki and Yoshida 1998; Morimura et al. 1998; Sato et al. 1998; Ito et al. 2000; Miyagi Cancer Society
2003). For example, according to reports from the Miyagi Prefecture Cancer Registry, the ageadjusted morbidity rate for intraepithelial carcinoma in women under 30 has increased over the last 20 years from 0.4 to 3.3 per 100000 women. Much research has identified that occurrence of cervical cancer is attributable to infection of the uterine cervix with human papillomavirus (HPV), and this infection route is known to be associated with sexual behavior (Yaegashi et al. 1989; Anttila et al. 1999; Yoshikawa et al. 1999; Woodman et al. 2001). It seems that recent changes in sexual behavior, such as decrease in age of first intercourse and an increase in the number of sexual partners, result in the spread of HPV infection among young women and lead to an increase in the cervical cancer morbidity rate among young people (Anttila et al. 1999). In the light of this, some countries have decided to begin cervical cancer screening at the age of 18 , while others have decided to begin it at the age of first intercourse (Dickinson 2002; Disaia and Creasman 2002). Regardless of the fact that the same social background exists in Japan, cervical cancer screening is restricted to women aged 30 or older. It may be an important reason why cervical cancer morbidity rate has remained high among women under the age of 30 in Japan.

The question to be asked, therefore, is what extent would abnormal cases be discovered if cervical cancer screening were conducted in Japan on young women under the age of 30 ? Unfortunately, sufficient data is not available on this because the screening subjects in Japan had been 30 years old or older. In other words, the 
current situation is that the facts concerning cervical cancer screening in women under the age of 30 in Japan are unknown.

On the other hand, a woman will most likely visit a gynecological care facility even under the age of 30 if there is possibility of pregnancy. It seems that such women often receive first medical consultation when they realize that they are pregnant and come to an obstetrics and gynecology department, and such an occasion is an opportunity for the care facility to conduct a cytologic examination in symptom-free women under the age of 30 .

Here, in order to survey the results of cervical cancer screening for all women under the age of 30 , we studied the results of cervical cytologic examination of pregnant women and the course of subjects with cytodiagnostic abnormalities.

\section{Materials and Methods}

Cervical cytologic examination was conducted, as screening for cervical cancer, in pregnant women who visited ten care facilities in Miyagi and Iwate prefectures (Tohoku University Hospital, Sendai National Hospital, Tohoku Kousai Hospital, Sendai Municipal Hospital, Sendai Red Cross Hospital, Furukawa Municipal Hospital, Sanuma Municipal General Hospital, Miyako Prefectural Hospital, Suzuki Hospital and Nakagawa Obstetrics and Gynecology Hospital) for pregnancy checkup over a ten-year period between 1993 and 2002. The cervical cytologic examination was conducted when the women underwent the initial pregnancy examination or during regular pregnancy checkups.

Subjects excluded from the study were women who were found to have cervical cytologic abnormalities before pregnancy and became pregnant during the follow-up period and women who were referred by other hospitals with cytologic abnormalities or pathological changes in the uterine cervix. The remaining 28616 women (including 538 puerperant women) were employed as subjects to form the pregnant women's group in the study. The age distribution in the pregnant women's group was 560 subjects aged 19 or younger, 4159 subjects aged between 20 and 24, 11034 subjects aged between 25 and 29, 9194 subjects aged 30 to 34,3265 subjects aged 35 to 39 and 404 subjects aged 40 or older, and the mean age was $29.0 \pm 4.88$ years. The group included 15753 subjects under the age of 30, which were $55.0 \%$ of the total. The cytologic evaluation was done based on the evaluation criteria of cervical cytologic examination of the Japanese Association of Obstetrics and Gynecology, and cases evaluated as Class IIIa, IIIb, IV or V were determined as cases requiring close examination (hereinafter, "required close examination"). The course of subjects who required close examination was followed up, and their histological diagnosis, method of treatment, and outcome were studied.

As control, 108289 women who were subjected to cervical cancer mass screening (hereinafter, "mass screening") in Miyagi Prefecture in 2001 were used as a mass screening group. The mean age was 54.8 years old.

The $\chi^{2}$ test was used to verify statistical difference. Because a relatively large number of subjects was studied, a significant difference was determined when the risk was $p<0.01$.

\section{Results}

\section{Rate of required close examination}

Three hundred and twenty-one subjects out of 28616 pregnant women group $(1.12 \%)$ were found to belong to class III or higher in cytology (Table 1). In the mass screening group, 907 subjects out of 108289 women $(0.84 \%)$ were in class III or higher in cytology. The rate in the pregnant women's group was significantly higher than that in the mass screening group $(p<0.001)$.

\section{Rate of required close examination by age stratification}

The subjects were divided into age groups of five years, and the rate of required close examination was studied by age group (Table 2). In the pregnant women's group, the rates of required 
Y. Abe et al.

TABLE 1. Cytological results for the pregnant women's group and mass screening group

\begin{tabular}{lcc}
\hline & Pregnant women's group & Mass screeinig group \\
\hline Cases (persons) & 28616 & 108289 \\
Close examination (persons) & 321 & 907 \\
Rate of close examination (\%) & 1.12 & 0.84 \\
& & \\
& & $p<0.001$ \\
Cytology & & 689 \\
IIIa & 236 & 190 \\
IIIb & 79 & 11 \\
IV & 6 & 17 \\
V & 0 & \\
\hline
\end{tabular}

close examinations were $2.32 \%$ in age group of 19 or younger, $1.80 \%$ in age 20 to $24,0.96 \%$ in age 25 to $29,0.94 \%$ in age 30 to $34,1.04 \%$ in age 35 to 39 and $1.73 \%$ in age 40 or older. The rate was significantly higher in age group of 19 or younger compared with the age group of 25 to 29 , 30 to 34 and 35 to 39 , respectively $(p<0.01$ ). The rate was also significantly higher in the women aged 20 to 24 than the women in the 25 to 29 and 30 to 34 age groups $(p<0.01)$, though there was no significant difference compared with women 35 years old or older. When the pregnant women's group was divided into two groups, of women under the age of 30 and women aged 30 or older, and the two groups were compared, the rate was $1.23 \%$ (194/ 15753$)$ in the group of women under the age of 30 and $0.99 \%$ (127/ 12863 ) in the group of women aged 30 or older. No statistically significant difference was found between them.

Next, we compared the pregnant women's group and the mass screening group. The rate of required close examination in the mass screening group was $1.78 \%$ for women aged 30 to $34,1.12 \%$ for women aged 35 to 39 and $0.91 \%$ for women aged 40 to 44 . When these rates were compared with the corresponding age groups in the pregnant women's group, the rate was significantly higher $(p<0.001)$ for the women aged 30 to 34 in the mass screening group. However, no statistically significant differences between the pregnant women's group and the mass screening group were found for the women aged 35 to 39 or for the women aged 40 or older. When groups were divided into two groups of women under the age of 30 and women aged 30 or older, the rate of required close examination in the pregnant women's group aged 30 or older had no significance compared with the corresponding age group in the mass screening group $(0.99 \%$ vs. $0.84 \%)$. The rate of required close examination in the pregnant women's group of younger than 30 years old was significantly higher than that in the mass screening group $(p<0.01)(1.23 \%$ vs. $0.84 \%)$.

\section{Follow-up of subjects requiring close examina- tion}

Followup was conducted on 321subjects who required close examination. Of these subjects, 60 could not be followed up because they changed hospitals, changed their address, and so on. Three cases of class IIIb and 57 cases of class IIIa were found. The remaining 261 were studied, and no abnormalities were found in close examination (spot histological examination) or repeated cytologic examination in 189 of these cases. Thirty-six cases in which atypical epitheliums were found are still under follow-up study. Two subjects were diagnosed in spot histological examination as having carcinoma in situ (CIS), but 
TABLE 2. The rate of close examination by age group

\begin{tabular}{|c|c|c|c|c|}
\hline \multirow[b]{2}{*}{ Ages } & \multicolumn{2}{|c|}{ Pregnant women's group } & \multicolumn{2}{|c|}{ Mass screening group } \\
\hline & Cases & $\begin{array}{l}\text { Rate of close examination } \\
(\%)\end{array}$ & Cases & $\begin{array}{c}\text { Rate of close examination } \\
(\%)\end{array}$ \\
\hline$\sim 19$ & 560 & $13(2.32) \square$ & & \\
\hline $20 \sim 24$ & 4,159 & \begin{tabular}{l|l}
$75(1.80)$ & -1.23
\end{tabular} & & \\
\hline $25 \sim 29$ & 11,034 & $106(0.96)-$ & & \\
\hline $30 \sim 34$ & 9,194 & $86(0.94)$ & 5,223 & $93(1.78) \square$ \\
\hline 35 39 & 3,265 & $34(1.04) \quad-0.99$ & 8,605 & $96(1.12)$ \\
\hline 40 44 & 404 & $7(1.73)$ & 11,477 & $105(0.91) \square$ \\
\hline
\end{tabular}

their whereabouts and the courses of their diseases are unknown. Thirty-four subjects are currently still under some kind of treatment, and the age, method of treatment and final histopathological diagnosis of these 34 cases are shown in Table 3. Seventeen of these subjects $(50 \%)$ were under the age of 30 . The method of treatment was conization in 25 cases, simple total hysterectomy in 7 cases, modified radical hysterectomy in 1 case, and radical hysterectomy in 1 case. The final histopathological diagnosis was moderate dysplasia in 2 cases, severe dysplasia in 10 cases, CIS in 19 cases, microinvasive carcinoma in 2 cases and invasive carcinoma in 1 case. The ages of all three cases of microinvasive and/or invasive carcinoma were 30 years old or younger $(23,23,27$ years old, respectively), and their cytologies were evaluated as class IIIb.

\section{Discussion}

There has been an increase in the incidence of cervical cancer among young women in the developed countries (Wright et al. 1994; Hamasaki et al. 1998; Morimura et al. 1998; Anttila et al. 1999; Ito et al. 2000). Very recently, the Ministry of Health, Labor and Welfare proposed that cervical cancer screening should be conducted for women aged 20 to 29 years old even in Japan. However, there are insufficient data available in Japan to predict the results of screening possibly conducted on women under the age of 30 . To the best of our knowledge, this is the first detailed re- port concerning about cervical cancer screening of symptom-free women under the age of 30 .

Cervical cancer screening conducted on healthy pregnant women revealed that the rate of required close examination in the pregnant women's group was even higher than in the mass screening group, consisting of women aged 30 or older who undergo this screening in compliance with the current Health and Medical Service Law for the Aged. Our data in the pregnant women's group indicated that the rate of required close examination was significantly higher in the women aged 24 or younger than in older women. It is known that while the morbidity rate of cervical cancer has been declining every year, the morbidity rate of women under the age of 30 alone has been rising in Japan (Sato et al. 1998; Miyagi Cancer Society 2003). We believe that establishment of a screening system for women under the age of 30 , who presently have the highest cervical cancer morbidity rate, will have the greatest impact on lowering the country's cervical cancer morbidity rate.

Previous reports concerning cervical cancer screening in pregnant women in Japan reported that the rate of required close examination was 1.2\% (Abe et al. 2004), and the results of this study seem to confirm this rate. However, research samples in such reports were extremely small, numbering from 241 to 9051, and included cases of cytologic abnormalities identified before pregnancy. Our study was a joint research of sev- 
TABLE 3. The follow-up results in therapy cases

\begin{tabular}{|c|c|c|c|c|}
\hline Case & Age & Cytology & Histology & Treatment \\
\hline 1 & 23 & IIIa & SD & Conization \\
\hline 2 & 27 & $\mathrm{IIIa} \rightarrow$ IV & CIS & Conization \\
\hline 3 & 27 & $\mathrm{IIIa} \rightarrow$ IV & CIS & Conization \\
\hline 4 & 28 & $\mathrm{IIIa} \rightarrow \mathrm{IIIb}$ & CIS & Conization \\
\hline 5 & 30 & $\mathrm{IIIa} \rightarrow \mathrm{IIIb}$ & SD & Conization \\
\hline 6 & 32 & $\mathrm{IIIa} \rightarrow \mathrm{IIIb}$ & SD & Conization \\
\hline 7 & 23 & IIIb & MIC & ATH \\
\hline 8 & 23 & IIIb & MIC & ATH \\
\hline 9 & 27 & IIIb & IC & $\mathrm{RH}+$ radiation \\
\hline 10 & 35 & IIIb & CIS & Conization \\
\hline 11 & 36 & IIIlb & CIS & Conization \\
\hline 12 & 36 & IIIb & CIS & Conization \\
\hline 13 & 34 & IV & CIS & Conization \\
\hline 14 & 19 & $\mathrm{IV} \rightarrow \mathrm{IIIb}$ & SD & Conization \\
\hline 15 & 26 & IV & CIS & ATH \\
\hline 16 & 30 & IV & CIS & VTH \\
\hline 17 & 34 & IV & CIS & Conization \\
\hline 18 & 26 & $\mathrm{IIIb} \rightarrow \mathrm{IV}$ & CIS & Conization \\
\hline 19 & 28 & IIIb & SD & Conization \\
\hline 20 & 29 & $\mathrm{IIIb} \rightarrow \mathrm{IV}$ & CIS & Conization \\
\hline 21 & 29 & $\mathrm{IIIb} \rightarrow \mathrm{IV}$ & CIS & Conization \\
\hline 22 & 32 & $\mathrm{IIIb} \rightarrow \mathrm{IV}$ & CIS & Conization \\
\hline 23 & 33 & IIIb & SD & Conization \\
\hline 24 & 34 & $\mathrm{IIIb} \rightarrow \mathrm{IV}$ & CIS & Conization \\
\hline 25 & 31 & IV & CIS & Conization \\
\hline 26 & 36 & IIIb & SD & ATH \\
\hline 28 & 25 & IIIa & SD & Conization \\
\hline 27 & 33 & IIIb & SD & Conization \\
\hline 29 & 31 & IIIb & MD & Conization \\
\hline 30 & 29 & $\mathrm{IIIa} \rightarrow \mathrm{IIIb}$ & MD & Conization \\
\hline 31 & 28 & IV & CIS & Conization \\
\hline 32 & 29 & $\mathrm{IIIa} \rightarrow \mathrm{IIIb}$ & CIS & ETH \\
\hline 33 & 39 & IIIb & CIS & ATH \\
\hline 34 & 31 & IIIa & SD & Conization \\
\hline
\end{tabular}

MD, moderate dysplasia; SD, severe dysplasia; CIS, carcinoma in situ; MIC, microinvasive carcinoma; IC, invasive carcinoma; ATH, abdominal total hysterectomy; VTH, vaginal total hysterectomy; ETH, extended total hysterectomy(modified radical hysterectomy); $\mathrm{RH}$, radical hysterectomy. 
eral facilities, ranging from general practitioner clinics to higher-order medical facilities, and was the largest-scale research with a subject population exceeding 28000 . The subjects in this research do not include all of the pregnant women who underwent cytologic examination but excluded patients for which cytologic abnormalities were identified before pregnancy. Therefore, this research reflects more accurately the results of cervical cancer mass screening of symptom-free subjects.

The rate of Japanese women who experience childbirth even once in their lives, as calculated from the rate of first childbirth for each age, is 64\% (Kaneda 2003). If cervical cancer screening were conducted whenever a woman came to a clinic or hospital for a physical checkup in pregnancy, about two thirds of the total population of women in Japan would undergo cervical cancer screening. In addition, it seems that many women under the age of 30 get their first chance to be checked in an obstetrics and gynecology department when they become pregnant. It therefore appears that pregnancy would be the best opportunity to introduce women for the first time to cervical cancer screening. In other words, cervical cancer screening of the pregnant women is a useful means to find cervical carcinoma in young women. We expect this research to change the age range of subjects for cervical cancer screening.

\section{Acknowledgments}

This study was intended and subsidized by the Miyagi chapter of Japan Obstetrician and Gynecologist Society, and was researched by Tohoku University's Department of Obstetrics and Gynecology, School of Medicine. We express our appreciation of the opportunity granted to us for this study.

We wish to thank Miyagi Prefecture Cancer Society, National Sendai Hospital, Tohoku Kousai Hospital, Sendai Municipal Hospital, Sendai Red Cross Hospital, Suzuki Hospital, Sanuma Hospital, and Nakagawa Obstetrics and Gynecology Hospital for their cooperation in this study.

\section{References}

Abe, Y., Okamura, C., Niikura, H., Terada, Y., Murakami, T., Ozawa, N., Nagai, H., Wada, Y., Uehara, S., Watanabe, T., Tanigawara, S., Wagatsuma, S., Yoshida, Y., Igarashi, T., Suzuki, M., Nakagawa, K., Ito, K., Okamura, K. \& Yaegashi, N. (2004) Study of cervical cytologic examination during physical checkup of pregnant women: Cervical cancer screening in women under the age of thirty. Tohoku Journal of Obstetrics and Gynecology, 51, 24-29. (in Japanese)

Anttila, A., Pukkala, E., Soderman, B., Kallio, M., Nieminen, P., Hakama, M. (1999) Effect of organised screening on cervical cancer incidence and mortality in Finland, 1963-1995: recent increase in cervical cancer incidence. Int. J. Cancer, 83, 59-65.

Dickinson, J.A. (2002) Cervical screening: time to change the policy. Med. J. Australia, 176, 547-550.

Disaia, P.J. \& Creasman, W.T. (2002) Preinvasive disease of the cervix. In: Clinical Gynecologic Oncology, P.J. Disaia \& W.T. Creasman, 6th ed, St. Louis, London, Philadelphia, Sydney, Toronto, Mosby, Inc., 1-2.

Hamasaki, C. \& Yoshida, K. (1998) Epidemiology and Prevention of Uterine Cancer. Obstet. Gynecol., 65, 1686-1690. (in Japanese)

Health and Welfare Statistics Association (2000) Report of senile health and welfare project by the division of statistics and information secretary of health and walfare. 328-333. (in Japanese)

Ito, T., Ishizuka, T., Suzuki, K., Ikoma, Y., Saito, J., Onuma, M., Miwa, T., Hashiba, Y., Kuno, N., Horibe, N., Mizuno, K., Ishikawa, K. \& Kazeto, S. (2000) Cervical cancer in young Japanese women. Arch. Gynecol. Obstet., 264, 68-70.

Japan Public Health Association (2001) A study by the Ministry of Health, Labour and Welfare. The efficacy evaluation of cancer screening, 7-9. (in Japanese)

Kaneda, I. (2003) Maternal and child health statistics of Japan. Mother's and Children's Health and Welfare Association, 48-50. (in Japanese)

Miyagi Cancer Society: Annual Report, 2002 (2003) Sendai, Japan, Miyagi Cancer Society.

Morimura, Y., Hoshi, K., Tomita, T., Hanyu, T., Sekimoto, S., Sato, A. (1998) A twenty-year period in mass screening for uterine cervical cancer in Fukushima Prefecture. Fukushima J. 
Med. Sci., 44, 59-64.

Sato, S., Matsunaga, G. \& Yajima, A. Mass screening for cancer of the uterine cervix in Miyagi Prefecture, Japan: Effects and problems. (1998) Acta Cytol., 42, 299-304.

Woodman, C.B., Collins, S., Winter, H., Bailey, A., Ellis, J., Prior, P., Yates, M., Rollason, T.P. \& Young, L.S. (2001) Natural history of cervical human papillomavirus infection in young women: a longitudinal cohort study. Lancet, 357, 1831-1836.

Wright, T.C., Kurman, R.J. \& Ferenczy, A.F. (1994) Precancerous lesion of the cervix. In: Blaustein's Pathology of the Female Genital Tract. 4th ed., edited by R.D. Kurman, NewYork, Ber- lin, Heidelberg, London, Paris, Tokyo, HongKong, Barcelona, Budapest, SpringerVerlag, pp. 229-277.

Yaegashi, N., Yajima, H., Shikano, K., Sato, S. \& Yajima, A. (1989) Detection of human papillomavirus type $52 \mathrm{~b}$ in cervical dysplastic tissue. Tohoku J. Exp. Med., 159, 215-219.

Yoshikawa, H., Nagata, C., Noda, K., Nozawa, S., Yajima, A., Sekiya, S., Sugimori H., Hirai, Y., Kanazawa, K., Sugase, M., Shimizu, H. \& Kawana, T. (1999) Human papillomavirus infection and other risk factors for cervical intraepithelial neoplasia in Japan. Br. J. Cancer, 80, 621-624. 